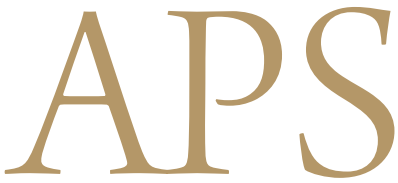

Archives of Plastic Surgery

\title{
Through Knee Amputation: Technique Modifications and Surgical Outcomes
}

\author{
Frank P Albino ${ }^{1}$, Rachel Seidel ${ }^{1}$, Benjamin J Brown ${ }^{1}$, Charles G Crone ${ }^{2}$, Christopher E Attinger ${ }^{1}$ \\ ${ }^{1}$ Department of Plastic Surgery, Georgetown University Hospital, Washington, DC; ${ }^{2}$ Nascott/Hanger Clinic, Washington, DC, USA
}

Background Knee disarticulations (KD) are most commonly employed following trauma or tumor resection but represent less than $2 \%$ of all lower extremity amputations performed in the United States annually. KDs provide enhanced proprioception, a long lever arm, preservation of adductor muscle insertion, decreased metabolic cost of ambulation, and an end weight-bearing stump. The role for KDs in the setting of arterial insufficiency or overwhelming infection is less clear. The purpose of this study is to describe technique modifications and report surgical outcomes following KDs at a high-volume Limb Salvage Center.

Methods A retrospective study of medical records for all patients who underwent a throughknee amputation performed by the senior author (C.E.A.) between 2004 and 2012 was completed. Medical records were reviewed to collect demographic, operative, and postoperative information for each of the patients identified.

Results Between 2004 and 2012, 46 through-knee amputations for 41 patients were performed. The mean patient age was 68 and indications for surgery included infection (56\%), arterial thrombosis (35\%), and trauma (9\%). Postoperative complications included superficial cellulitis (13\%), soft tissue infection (4\%), and flap ischemia (4\%) necessitating one case of surgical debridement (4\%) and four trans-femoral amputations (9\%). 9 (22\%) patients went on to ambulate. Postoperative ambulation was greatest in the traumatic cohort and for patients less than 50 years of age, $\mathrm{P}<0.05$. Alternatively, diabetes mellitus and infection reduced the likelihood of postoperative ambulation, $\mathrm{P}<0.01$.

Conclusions Knee disarticulations are a safe and effective alternative to other lower extremity amputations when clinically feasible. For patient unlikely to ambulate, a through-knee amputation maximizes ease of transfers, promotes mobility by providing a counterbalance, and eliminates the potential for knee flexion contracture with subsequent skin breakdown.

\section{Keywords Amputation / Disarticulation / Extremity}

Correspondence:

Christopher E Attinger

Department of Plastic Surgery, 3800

Reservoir Road NW, Washington,

DC 20007, USA

Tel: +1-202-444-8751

Fax: +1-202-444-0300

E-mail: cattinger@aol.com

No potential conflict of interest relevant to this article was reported.

\section{INTRODUCTION}

Functional outcomes are, on average, superior following below the knee amputations (BKAs) compared to above the knee amputations (AKAs) [1-6]. Consequently, trans-tibial amputations are preferred when clinically feasible based upon location of injury and lower extremity perfusion. In the event of a failed BKA, or in the setting of one that is likely to fail, the femur is often regarded as the next "level" at which to amputate. Despite potentially more reliable healing, AKAs require almost twice as much 
energy expenditure for ambulation compared to BKAs, are not end-weight bearing, and result in fewer ambulatory patients $[5,7,8]$. Thirty to $50 \%$ more energy is required to ambulate following an AKA compared to a BKA $[7,8]$. Additionally, walking speeds are reduced while energy demands are increased due to heavy prosthetics and two artificial joints. Given these undesirable factors, AKAs may not be the most appropriate level of amputation to improve functional outcomes and preserve limb length in the event that a BKA is not feasible.

Knee disarticulations (KD) represent less than $2 \%$ of all lower extremity amputations performed in the United States annually [1]. KDs provide an end weight bearing stump, have enhanced proprioception, preserve adductor muscle insertion, have a longer lever arm, and decrease the metabolic cost of ambulation [9]. Yet despite these proven benefits, the $\mathrm{KD}$ is frequently overlooked as a surgical option with concerns for a bulky distal stump, prosthetic knee that is distal to the contralateral normal leg, or healing complications from longer soft tissue flaps. The Mazet technique has previously addressed concerns of a bulky stump by shaving the femoral condyles and excising the patella to make a conical distal amputation [9]. For a patient unlikely to ambulate, a through-knee amputation maximizes ease of transfers, promotes mobility by providing a better counterbalance, and eliminates the potential for knee flexion contracture with subsequent distal stump skin breakdown. The purpose of this manuscript is to describe a single surgeon's experience with the through-knee amputation at a high-volume Limb Salvage Center in order to identify appropriate candidates for a knee disarticulation, provide surgical technique modifications aimed at improv-

\section{Fig. 1. Knee disarticulation case}

A 57-year-old female with history of diabetes mellitus, peripheral vascular disease, hypertension, and coronary artery disease presents with calcaneal wound notable for critical stenosis of anterior tibial, posterior tibial, and peroneal vessels. The tibial tuberosity is marked along with the skin incision for a planned through knee disarticulation.

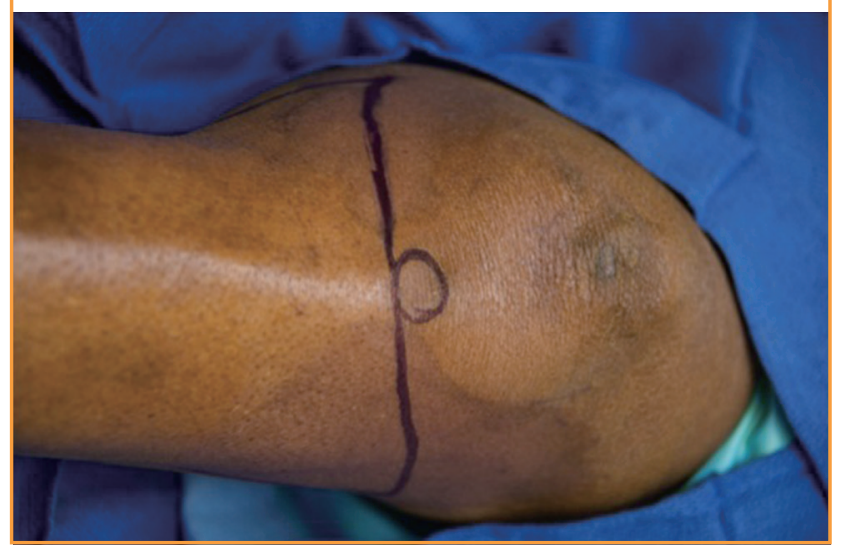

ing postoperative function, and discuss patient outcomes.

\section{METHODS}

An institutional review board approved, retrospective study of medical records for all patients who underwent a through-knee amputation performed by the senior author (C.E.A.) between 2004 and 2012 was completed. Medical records were reviewed to collect demographic, operative, and postoperative information for each of the patients identified. In the setting of a staged procedure, only patients who underwent a completion knee disarticulation were included. Patients were excluded if they underwent a through-knee amputation as a first stage procedure to a planned AKA. Inpatient and outpatient clinic records were reviewed to obtain information regarding medical co-morbidities, previous surgical procedures, indications for surgery, operative techniques, minor complications managed as an outpatient, major complications requiring reoperation, time to healing, prosthetic fittings, stump discomfort, and ambulatory status. A twotailed Fishers exact or chi-square test (VassarStats, Copyright 1998-2011 Richard Lowry) was used to determine statistical relevance, a P-value less than or equal to 0.05 was considered significant.

\section{Surgical technique}

The leg is marked at the level of the knee with a posterior soft tissue flap of sufficient length to close the incision without tension (Figs. 1, 2). A proximal thigh tourniquet is placed, the patient is prepped and draped, planned incisions are re-marked, and the tourniquet is inflated without exsanguination. The incisions are sharply carried down circumferentially to the level of the knee, through the joint, and along the drawn posterior flap (Figs. 3, 4). The distal leg is removed from the operative field (Fig. 5). The distal end of the femur is sharply shaved down from distal to proximal using a sagittal saw anteriorly, posteriorly, medially, and

\section{Fig. 2. Intraoperative markings}

Knee disarticulation skin incision marking.

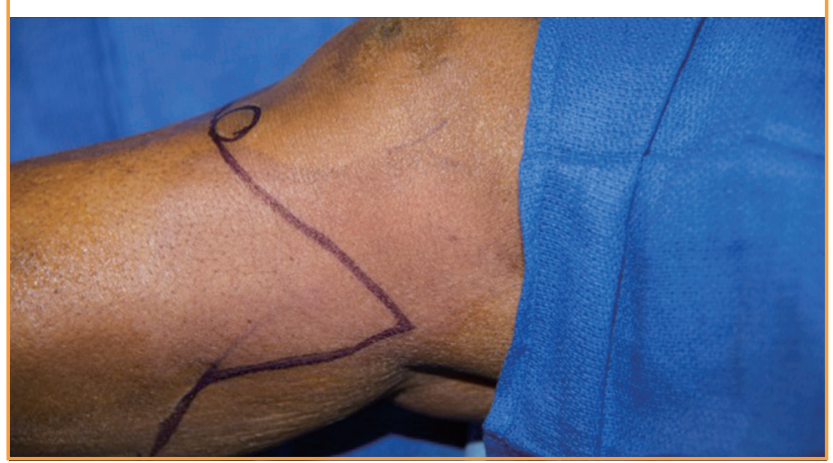




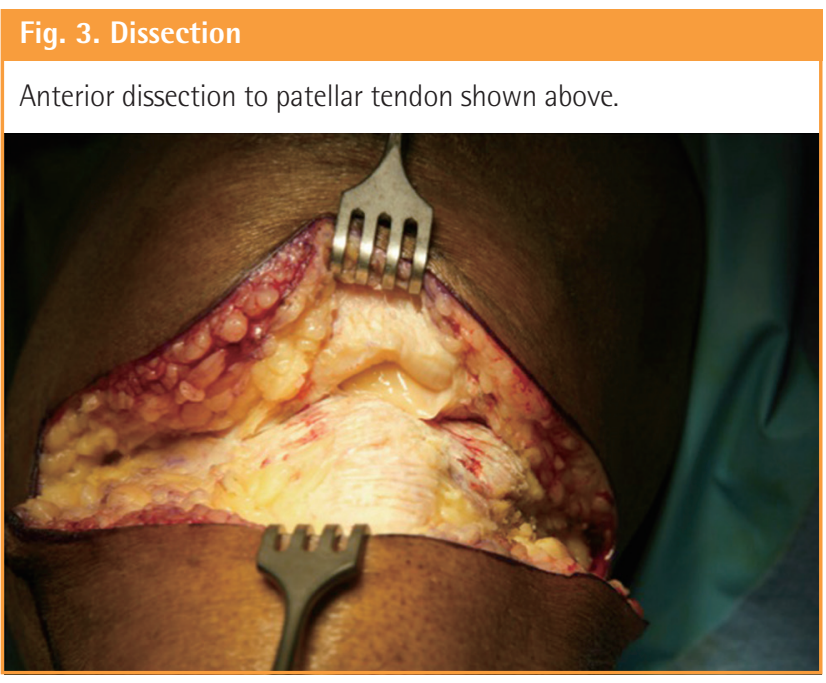

\section{Fig. 4. Posterior flap}

The incisions are sharply carried down circumferentially to the level of the knee and along the drawn posterior flap.

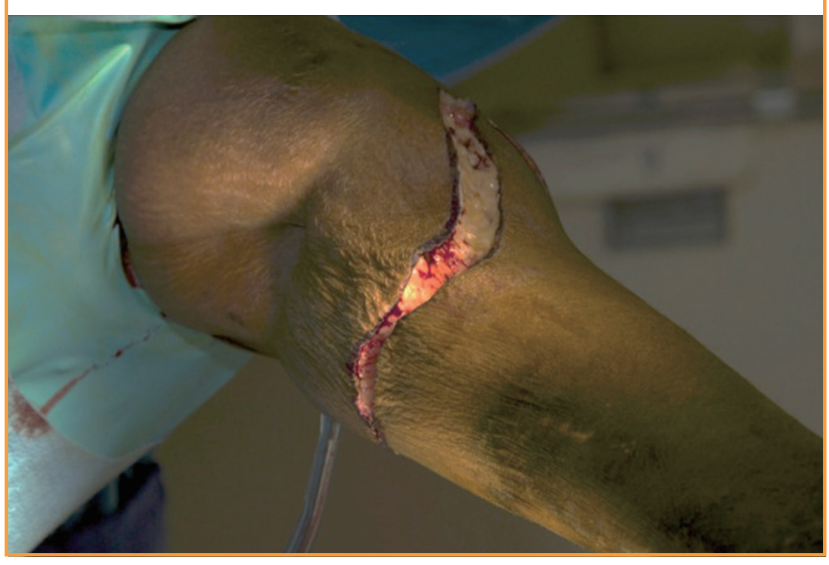

\section{Fig. 5. Disarticulation}

The distal leg is removed from the operative field with ligation of the popliteal vessel.

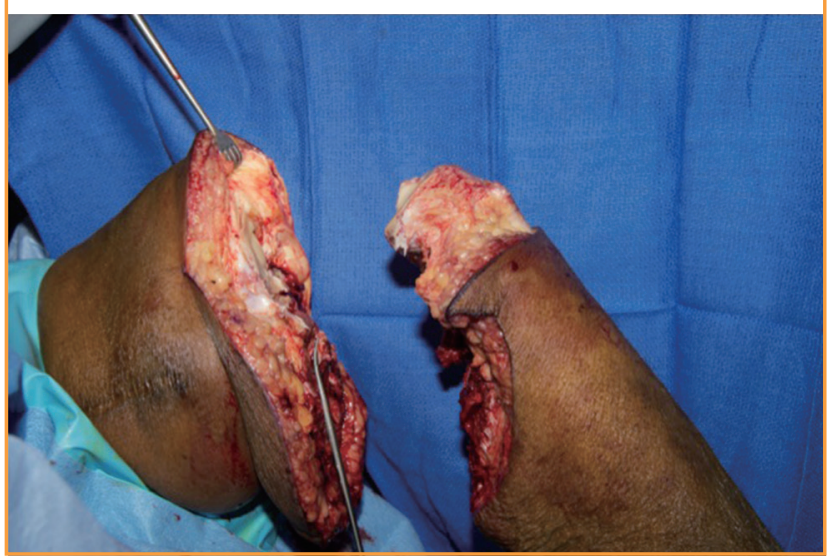

laterally converting the condyles into a "box" (Figs. 6, 7). Great care is taken not to disturb the insertion of the Adductor Mag-
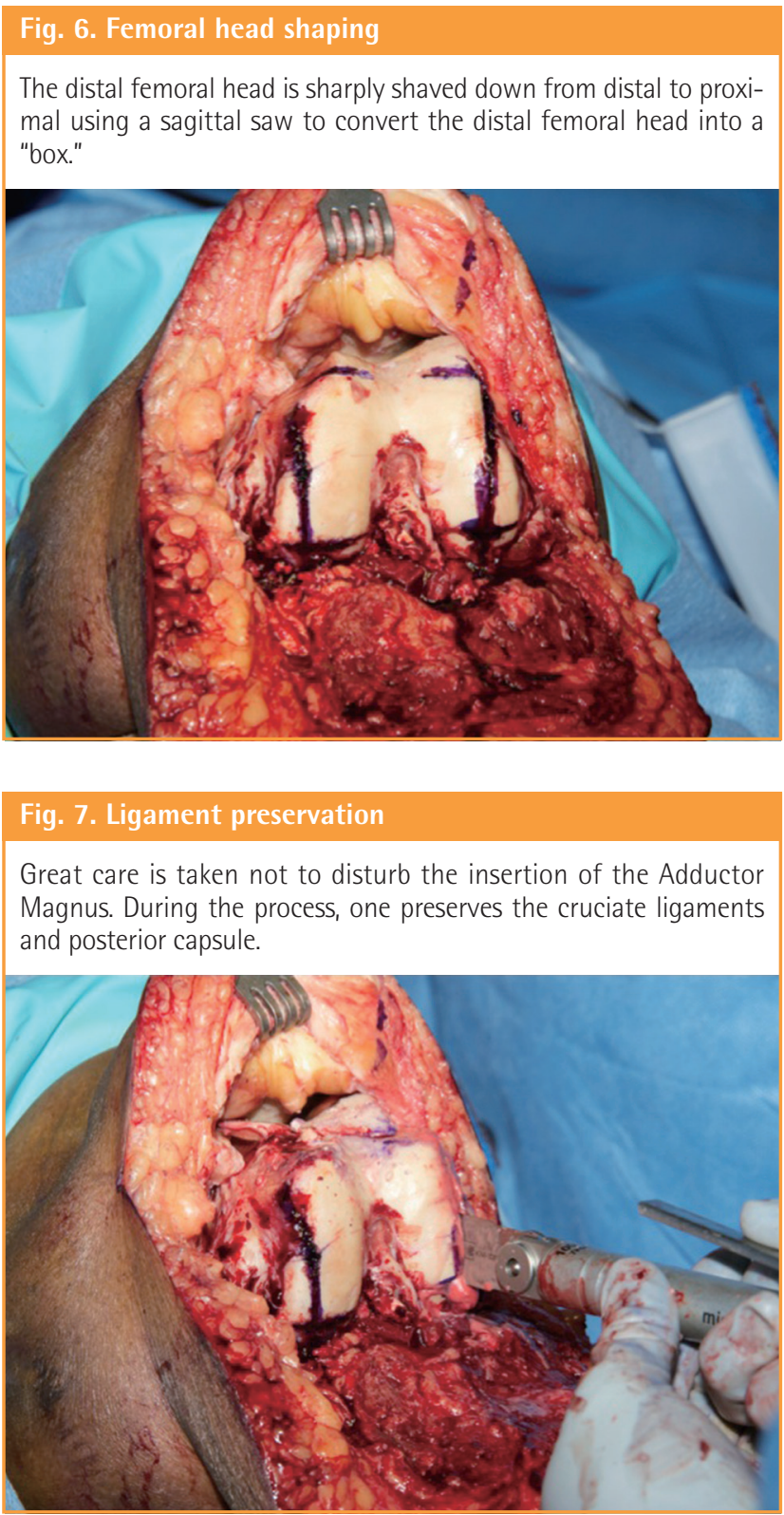

nus. During the process, the cruciate ligaments and posterior capsule are preserved. The patella is identified and the posterior articulating surface is shaved to cancellous bone (Fig. 8) The limb is then irrigated sufficiently with normal saline and the surgical gloves and instruments are changed for a clean, post-debridement set as fresh sterile drapes are placed on the field.

The patella is advanced to cover the distal surface of the femur as an end-weight bearing surface. The quadriceps tendon is sewn to the preserved cruciate ligament and posterior joint capsule so that the posterior surface of the patella is in direct contact with the distal end of the femur (Figs. 9, 10). Next, the hamstring muscles are myodesed to the quadriceps tendon and posterior capsule using 2.0-polydioxanone (PDS) so that the distal femur is completely covered. Lastly, a Jackson Pratt drain is 


\section{Fig. 8. Patella advancement}

The patella is identified and the posterior articulating surface is shaved to cancellous bone. The patella is advanced to cover the distal surface of the femur as an end-weight bearing surface.

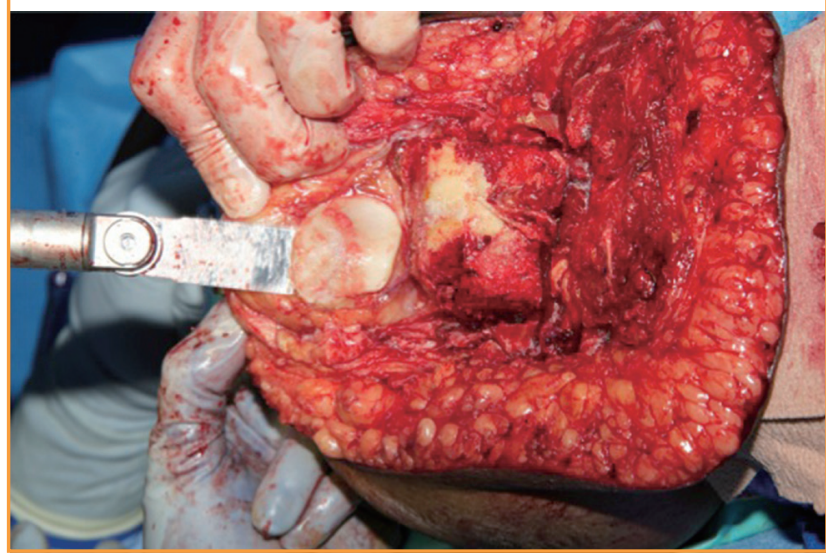

\section{Fig. 9. Patella cap}

The quadriceps tendon is sewn to the preserved cruciate ligament and posterior joint capsule so that the posterior surface of the patella is in direct contact with the distal end of the femur.

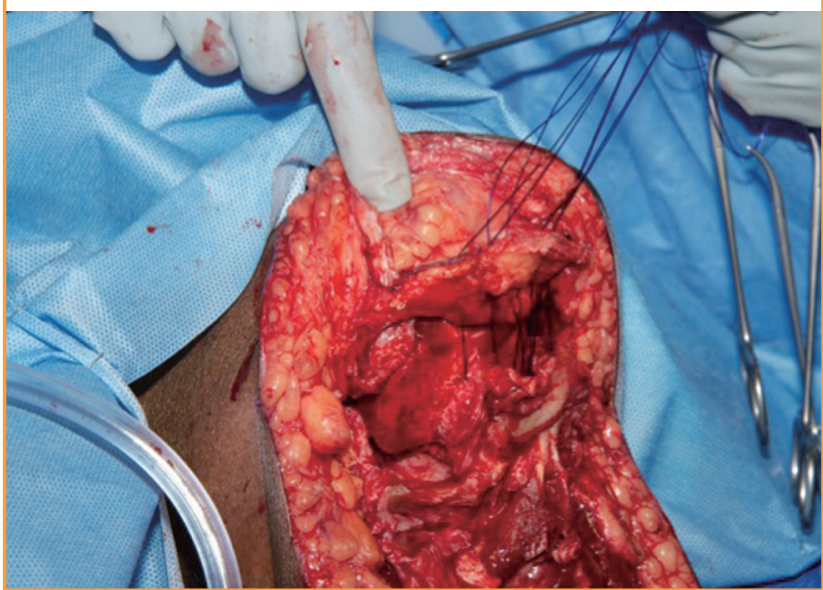

placed over the myodesis and the posterior flap is trimmed so that it can be sewn to the anterior flap without dog ears using 2.0-prolene vertical mattress suture. The drain is removed when daily output is less than $30 \mathrm{~mL}$ and the external sutures are removed after two weeks (Fig. 11).

In the setting of infection, the disarticulation is staged to prevent possible contamination of the definitive amputation. During the first procedure, the level of infection is clinically evaluated and a trans-tibial amputation is carried out distal to the planned posterior flap but proximal to the infected tissue. Intraoperative cultures are obtained and the wound bed is irrigated sufficiently with normal saline. Finally, a negative pressure wound vacuum assisted closure dressing is applied. After 2 to 3 days when the antibiotic regimen has been targeted to the finalized culture data, the patient returns to the operating room for a formal knee disarticulation as outlined above. In the setting of

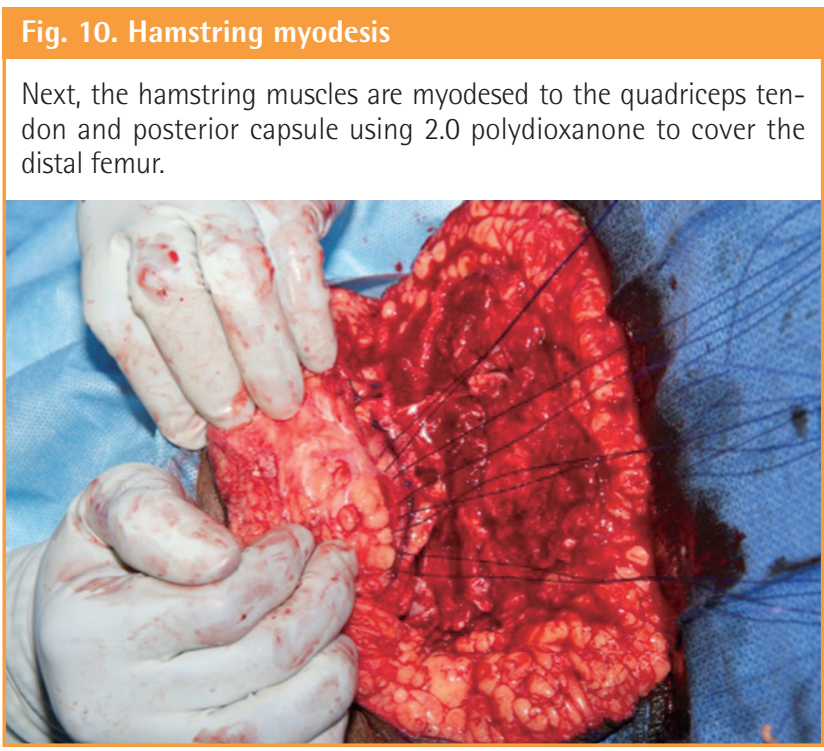

Fig. 11. Postopertiave evaluation

One year postoperative result following left knee disarticulation demonstrating a well-healed incision $(A, B)$ with corresponding lateral $\mathrm{X}$-ray $(\mathrm{C})$.
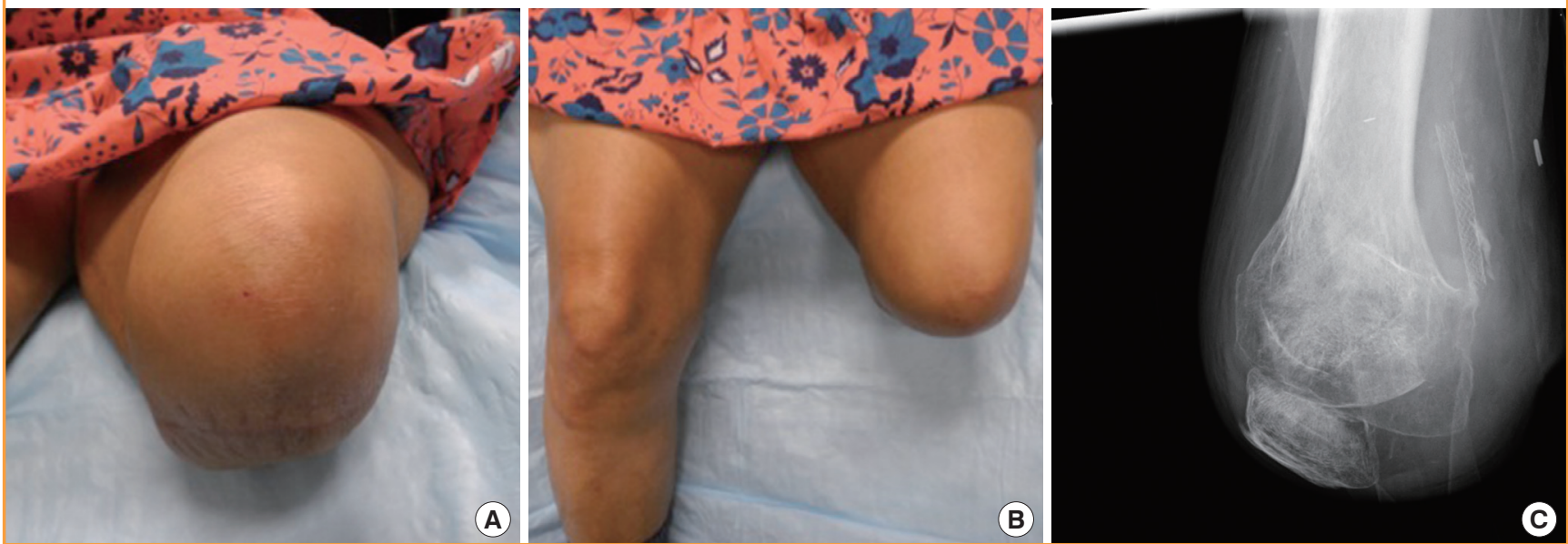
an ischemic limb and in the absence of infection, this procedure is performed in a single stage.

\section{RESULTS}

Between 2004 and 2012, the senior surgeon (C.E.A.) performed 46 through-knee amputations for 41 patients. The mean age at the time of surgery was 68 years (range, 22-99 years); $44 \%$ of patients $(18 / 41)$ were male while $56 \%$ of patients (23/41) were female. The right lower extremity was affected in $41 \%$ (19/46) of cases while 59\% (27/46) of knee disarticulations were performed on the left lower extremity. Thirteen patients (32\%) had not ambulated within 6 months prior to surgery. Patient co-morbidities included hypertension 80\% (33/ 41 ), peripheral vascular disease $73 \%$ (30/41), diabetes mellitus $70 \%$ (29/41), coronary artery disease 54\% (22/41), renal insufficiency $46 \%$ (19/41), congestive heart failure 32\% (13/41), history of a myocardial infarction 17\% (7/41), stroke 17\% (7/ 41 ), and dialysis dependence for end stage renal disease $12 \%$ (5/41) (Table 1). Seven patients had a history of tobacco use; three patients reported smoking within 3 months of surgery while four patients had previously quit tobacco use.

Fifty-six percent $(26 / 46)$ of knee disarticulations were performed primarily for an infection that had failed medical management. Lower extremity arterial thrombosis, 35\% (16/46), and trauma, 9\% (4/46), were other reasons for limb amputation. Aside from a higher incidence of diabetes mellitus in the infection cohort of patients $(\mathrm{P}=0.001)$, all other patient demographics were similar between patients who received a $\mathrm{KD}$ for infection, arterial thrombosis, or trauma (Table 1). In the setting of infection, intraoperative cultures were able to isolate a pathogen in $87 \%$ (22/26) of cases. Methicillin-Resistant Staphylococcus aureus (MRSA) was the most frequently isolated organism within this population (Table 2). The average patient had previously undergone 1.8 amputations (forefoot or trans-tibial) prior to a knee disarticulation. In total, $57 \%$ of knee disarticulations $(26 / 46)$ were preceded by an ipsilateral BKA; $85 \%$ in the infection cohort (22/26), 25\% within the ischemic population (4/ $16)$, and $0 \%$ of the trauma patients $(0 / 4), \mathrm{P}=0.002$.

Twenty-four KDs were performed in a single stage (52\%) while 22 cases (48\%) required two stages in the setting of infection with 4.5 days between procedures (range, 2 to 13), Table 3. While the majority of amputations for refractory infection were performed in two stages, two patients were treated in a single stage based on intraoperative clinical evaluation including loca-

Table 2. Identified microorganisms for infected wounds

\begin{tabular}{|ll|}
\hline Characteristic & \\
\hline Evidence of clinical infection (\%, n/total) & $57(26 / 46)$ \\
Microorganism identified (\%, n/total) & $85(22 / 26)$ \\
No organism growth on culture (\%, n/total) & $15(4 / 26)$ \\
Methicillin-resistant Staphylococcus aureus & 6 \\
Pseudomonas aeruginosa & 4 \\
Klebsiella pneumoniae & 3 \\
Proteus mirabilis & 2 \\
Escherichia coli & 2 \\
Streptococcus viridans & 1 \\
Enterobacter cloacae & 1 \\
Corynebacterium diphtheriae & 1 \\
Methicillin-sensitive Staphylococcus aureus & 1 \\
Enterococcus faecalis & 1 \\
\hline
\end{tabular}

\section{Table 1. Patient demographics}

\begin{tabular}{|c|c|c|c|c|c|}
\hline Medical co-morbidities & $\begin{array}{c}\text { Total patient } \\
\text { population }(n=41)\end{array}$ & $\begin{array}{c}\text { Infectious } \\
\text { etiology }(n=26)\end{array}$ & $\begin{array}{c}\text { Ischemic } \\
\text { etiology }(n=16)\end{array}$ & $\begin{array}{c}\text { Traumatic } \\
\text { etiology }(n=4)\end{array}$ & P-value \\
\hline Number of patients & 41 & - & - & - & - \\
\hline Number of KD & 46 & - & - & - & - \\
\hline Average age (yr) & 68 (range, 22-99) & - & - & - & - \\
\hline Infectious etiology & $56(26 / 46)$ & - & - & - & - \\
\hline Ischemic etiology & $35(16 / 46)$ & - & - & - & - \\
\hline Traumatic etiology & $9(4 / 46)$ & - & - & - & - \\
\hline HTN & $80(33 / 41)$ & $73(19 / 26)$ & $88(14 / 16)$ & $25(1 / 4)$ & 0.44 \\
\hline Peripheral vascular disease & $73(30 / 41)$ & $62(16 / 26)$ & $88(14 / 16)$ & 0 & 0.09 \\
\hline Diabetes mellitus & $70(29 / 41)$ & $92(24 / 26)$ & $31(5 / 16)$ & 0 & 0.001 \\
\hline Coronary artery disease & $54(22 / 41)$ & $46(12 / 26)$ & $63(10 / 16)$ & 0 & 0.35 \\
\hline Congestive heart failure & $32(13 / 41)$ & $35(9 / 26)$ & $25(4 / 16)$ & 0 & 0.73 \\
\hline H/O myocardial infarction & $17(7 / 41)$ & $8(2 / 26)$ & $31(5 / 16)$ & 0 & 0.08 \\
\hline Stroke & $17(7 / 41)$ & $12(3 / 26)$ & $25(4 / 16)$ & 0 & 0.40 \\
\hline End stage renal disease & $12(5 / 41)$ & $4(1 / 26)$ & $25(4 / 16)$ & 0 & 0.06 \\
\hline
\end{tabular}


tion of infected tissue and quality of proximal tissue. One patient died 11 days following surgery secondary to a myocardial infarction and cardiogenic shock. The average hospital stay was 9 days (range, 5 to 32 days) and the mean follow-up time as an outpatient was 311 days (range, 56 to 1332 days). Postoperative complications included superficial cellulitis $(13 \%, 4 / 46)$, soft tissue infection $(4 \%, 2 / 46)$, and flap ischemia $(4 \%, 2 / 46)$. These complications necessitated one case of surgical debridement (4\%) 15 days following the initial $\mathrm{KD}$ and four AKAs (9\%). These trans-femoral amputations occurred on average 44 days from the through-knee amputation (range, 6 to 113 days) for deep infection (2 patients) and critical limb ischemia (2 patients). There were no cases of a clinically significant seroma or hematoma formation postoperatively (Fig. 11). Two patients $(5 \%, 2 / 41)$ developed a painful neuroma and required subsequent nerve stump revision and implantation into muscle within 18 months from surgery. Both of these patients achieved pain relief without developing additional painful neuromas.

Ten patients (24\%) were fitted for a prosthetic device and ultimately 9 patients (22\%) ambulated with their prosthesis. When comparing patient factors or treatment variables the reason for needing an amputation, patient age, and ambulatory status were

\section{Table 3. Operative factors and complications}

\begin{tabular}{|lc|}
\hline Factor & $\%$ (n/total) \\
\hline Operative variables & \\
Single stage KD & $52(24 / 46)$ \\
Two-stage KD & $48(22 / 46)$ \\
Time between stages (day) & $4.5($ range, 2 to 13) \\
Complications & \\
30-day mortality & $2(1 / 41)$ \\
1-year mortality & $37(15 / 41)$ \\
Superficial cellulitis & $13(6 / 46)$ \\
Deep soft tissue infection & $4(2 / 46)$ \\
Incision/flap ischemia & $4(2 / 46)$ \\
Subsequent interventions & \\
Surgical debridement & $2(1 / 46)$ \\
Revision as AKA & $9(4 / 46)$ \\
Nerve decompression & $4(2 / 46)$ \\
\hline KD, knee disarticulation; AKA, above the knee amputations. \\
\hline
\end{tabular}

found to significantly affect outcomes (Tables 4, 5). Patients who received a $\mathrm{KD}$ following trauma were more likely to ambulate following their amputation $(100 \%, 4 / 4)$ than patients in the ischemic $(31 \%, 5 / 16)$ or infection cohorts $(0 \%, 0 / 26), \mathrm{P}=0.01$. Similarly, patients less than 50 years of age were also more likely to ambulate than patients over 50 years of age $(\mathrm{P}=0.01)$. Lastly, patients who did not ambulate prior to their $\mathrm{KD}$ were more likely to develop a postoperative complication, require additional surgical procedures, and less likely to ambulate following their amputation $(\mathrm{P}=0.02,0.01$, and 0.03 , respectively). Patient factors were assessed relative to ambulation following KDs, Table 6. Patients were less likely to ambulate following their amputation if they had diabetes mellitus ( $\mathrm{DM}, \mathrm{P}=0.002$ ) or the $\mathrm{KD}$ was performed to treat an infected wound $(\mathrm{P}=0.0001)$. On the other hand, they were more likely to ambulate if they ambulated prior to surgery $(\mathrm{P}=0.05)$ or required the amputation secondary to trauma $(\mathrm{P}=0.0009)$. There were no other detectable differences in outcomes based upon BMI, medical co-morbidities, smoking status, or length of hospital stay (P-value $>0.05$ ).

\section{DISCUSSION}

Greater than $80 \%$ of lower extremity amputations are performed for dysvascular extremities in the setting of peripheral vascular disease or diabetes mellitus [3]. Despite this, outcomes following knee disarticulations in the current literature are largely limited to traumatic injuries or oncologic resections. Surgical outcomes in other patient populations remain unclear. This

Table 4. Outcomes relative to reason for amputation

\begin{tabular}{|lcccc|}
\hline Outcome & $\begin{array}{c}\text { Trauma } \\
\text { (4) }\end{array}$ & $\begin{array}{c}\text { Ischemia } \\
\text { (16) }\end{array}$ & $\begin{array}{c}\text { Infection } \\
\text { (26) }\end{array}$ & P-value \\
\hline Any complication $(n=11)$ & 0 & 2 & 9 & 0.16 \\
Cellulitis $(n=6)$ & 0 & 2 & 4 & 1.0 \\
Soft tissue infection $(n=2)$ & 0 & 0 & 2 & 0.51 \\
Flap ischemia $(n=2)$ & 0 & 2 & 0 & 0.13 \\
Surgical revision $(n=5)$ & 0 & 1 & 4 & 0.63 \\
Achieved ambulation $(n=9)$ & 4 & 5 & 0 & 0.01 \\
\hline
\end{tabular}

Table 5. Outcomes relative to age or prior ambulation status

\begin{tabular}{|c|c|c|c|c|c|c|}
\hline Outcome & $\begin{array}{c}\text { Age }<50 \\
(n=7)\end{array}$ & $\begin{array}{c}\text { Age }>50 \\
(n=34)\end{array}$ & P-value & $\begin{array}{l}\text { Ambulatory within } \\
6 \mathrm{mo}(\mathrm{n}=28)\end{array}$ & $\begin{array}{c}\text { Non-ambulatory within } \\
6 \mathrm{mo}(\mathrm{n}=13)\end{array}$ & P-value \\
\hline Any complication $(n=11)$ & 1 & 10 & 0.65 & 4 & 7 & 0.02 \\
\hline Cellulitis $(n=6)$ & 1 & 5 & 1.0 & 4 & 2 & 1.0 \\
\hline Soft tissue infection $(n=2)$ & 0 & 2 & 1.0 & 1 & 1 & 0.53 \\
\hline Flap ischemia $(n=2)$ & 0 & 5 & 0.57 & 0 & 2 & 0.09 \\
\hline Surgical revision $(n=5)$ & 0 & 5 & 0.57 & 0 & 5 & 0.01 \\
\hline Achieved ambulation $(n=9)$ & 7 & 2 & 0.01 & 9 & 0 & 0.03 \\
\hline
\end{tabular}


Table 6. Factors predicting postamputation ambulation

\begin{tabular}{lccc|}
\hline Factor & $\begin{array}{c}\text { Ambulated } \\
\text { with KD } \\
(\mathbf{n}=\mathbf{9})\end{array}$ & $\begin{array}{c}\text { Non- } \\
\text { ambulatory } \\
(\mathbf{n}=\mathbf{3 2})\end{array}$ & P-value \\
\hline Patient co-morbidities & 6 & 27 & 0.60 \\
$\quad$ HTN & 5 & 25 & 0.44 \\
$\quad$ Peripheral vascular disease & 2 & 27 & 0.002 \\
Diabetes mellitus & 3 & 19 & 0.36 \\
Coronary artery disease & 1 & 12 & 0.37 \\
Congestive heart failure & 0 & 7 & 0.30 \\
Stroke & 0 & 5 & 0.49 \\
End stage renal disease & 9 & 19 & 0.05 \\
Ambulated within 6 months & & & \\
Etiology for amputation & 4 & 0 & 0.0009 \\
Trauma & 5 & 11 & 0.44 \\
Vascular insufficiency & 0 & 26 & 0.0001 \\
$\quad$ Infection & & & \\
Postoperative factors & 1 & 10 & 0.46 \\
Any complication & 1 & 5 & 0.75 \\
Cellulitis & 0 & 2 & 0.45 \\
Soft tissue infection & 0 & 5 & 0.51 \\
Flap ischemia & 0 & 5 & 0.51 \\
Surgical revision & & & \\
\hline KD, knee disarticulation; HTN, hypertension. & & \\
\hline
\end{tabular}

manuscript provides the first discussion of KDs in the treatment of patients with vascular compromise and one of the only applications of through-knee amputations for the treatment of diabetic wounds. These patient populations are of particular interest to optimize outcomes and restore function given high rates of re-amputation and significant morbidity. Twenty percent of patients with diabetes mellitus require multiple amputations and the mortality rates following lower extremity amputations approach $50 \%$ at 5 years [10-15]. It is important to determine role of through-knee amputations in complicated patients with multiple medical comorbidities to maximize post-operative function, improve quality of life, and minimize morbidity.

Attinger and Brown [11] added further confirmation to the importance of limb preservation in a series of 937 ambulatory, diabetic patients. Follow-up studies at this center demonstrate $77 \%$ of patients were ambulatory following a BKA, $24 \%$ required a surgical debridement, and $2 \%$ were ultimately converted to a trans-femoral amputation [12-14]. Comparing our study of knee disarticulations to this previous work, ambulation appears more common following BKAs (77\%) compared to KDs (22\%); however, these populations are not matched for demographic, pre-ambulation data or treatment variables. Variances in these ambulation rates likely reflect differences in expected outcomes between these surgical procedures and also in the patient populations treated. Importantly, 32\% of patients in our population were previously non-ambulatory prior to undergoing their knee disarticulation. Based upon the retrospective design of this study it is difficult to determine the exact cause of this limited mobility preoperatively. We suspect these patients were non-ambulatory secondary to overall decompensated health given the high percentage of medical co-morbidities and number of patients treated for advanced infections (57\%). The senior author does prefer the $\mathrm{KD}$ to above knee amputations whenever possible irrespective of ambulatory status because of its extended lever arm, improved counterbalance, and end bearing properties. In a recent study of 80 patients including 71 unilateral knee disarticulations and 9 bilateral amputations, Ten Duis et al. [6] found 52\% of patients were alive at 1 -year postoperatively, $28 \%$ suffered a wound healing complication, $34 \%$ regularly ambulated with the assistance of a prosthetic device, and $12 \%$ ultimately required an AKA. For comparison, our study resulted in an overall survival of $63 \%$ at 1 -year, wound healing complication in $24 \%(11 / 46)$ of amputations, ambulation in $22 \%$ (9/41) of patients, and proximal amputation rate of $9 \%(4 / 46)$.

There are a number of advantages to the knee disarticulation compared to a trans-femoral or trans-tibial amputation depending upon a patient's ambulatory status. For those patients who achieve ambulation, the KD provides an end-weight bearing amputation with preservation of the adductor musculature to improve thigh motion for ambulation, decrease energy expenditure, and simplify the creation of a prosthetic device. On the other hand, for patient unlikely to ambulate, a through-knee amputation maximizes ease of transfers because it is endbearing, promotes mobility using a wheelchair by providing better counterbalance, and eliminates the potential for a knee flexion contracture leading to subsequent distal stump skin breakdown. The use of a patella cap over the end of the femur has been previously described [15]; however, the technique described here adds further modifications to move the incision line anteriorly with a wellperfused posterior flap, preserve muscle attachments including the insertion of the adductor magnus, and staging the procedure in the setting of infection to further maximize the advantages of a knee disarticulation. The posterior raw surface of the patella cap is thought to form a bone union with the intercondylar raw surface of the femur; however, X-rays are not routinely obtained to support this prediction. Clinically, the patella remains palpable at the distal limb without evidence of anterior migration.

From a prosthetic fitting standpoint there are occasions when it's beneficial to preserve the solid base of the patella. The kneecap appears to form an anatomical lock around the distal aspect of the femur and palpates to form around the distal condyles of the femur. The tendon insertions are therefore protected from potential migration of the femur alone with the absence of the patella. If the patella is not structurally viable, it should be re- 
moved. The minimal addition of length from preserving the patella is worth the sacrifice as long as a thorough pre-amputation consult is performed with the patient and family prior to the procedure. If the patient is not a candidate for a prosthesis, then there is the added benefits for weight bearing with the patella during transferring and ambulating on the patient's knees or bilateral knee disarticulation amputations.

The need for a thorough discussion with the patient, family, and caregiver(s) is critical to determine what the pre and post amputations goals and expectations are following the most medically appropriate surgery option. The question of whether a short transtibial, knee disarticulation, or transfemoral amputation is the 'best' option must be evaluated by all involved. If the lever arm of the residual tibia is too short and there is a decision to attempt a transtibial level just to maintain the knee joint, the result may be more knee joint instability with minimal surface area to distribute the weight bearing in the socket interface. A higher level of amputation allows for additional alignment and prosthetic component options. This will benefit the patient who has a higher potential of wearing a prosthesis after surgery.

In the case of opting for a knee disarticulation level, there is the ability to distribute the weight bearing over a greater surface area. The patient has a better feel of his leg position with the thigh intact. The distal patella can give the sensation of having a knee joint. Without a prosthesis, the $\mathrm{KD}$ has extended lever arm, improved counterbalance and end bearing properties. A higher level of amputation (transfemoral, AKA) to allow for additional alignment and prosthetic component options may need to be considered if there are complications after the initial knee disarticulation surgery. The patient may then benefit from less surface area to weight bear and loss of end bearing capacity with a revision to a transfemoral amputation given a shorter lever arm. The extra room afforded by the loss of the femoral head allows for better alignment, rotation, and/or shock absorber adapters that often compensate for the need to load the residual limb more aggressively in the proximal aspect when revised to the AKA.

Knee disarticulations clearly have advantages over other lower extremity amputations and the important factor is to determine who will benefit most. Taylor et al. [13] identified a number of risk factors to predict patients who fail to ambulate following a through-knee amputation. Specifically, amputees were 10-times less likely to use a prosthetic if they were non-ambulatory prior to surgery. Additionally, age over 60 (Relative Risk 2.7) and coronary artery disease (RR 2.0) were identified as independent, negative predictors of post-amputation ambulation. Similarly, our study identifies pre-amputation ambulation, diabetes mellitus, and infection as significant barriers to postoperative ambulation. As a retrospective study of 46 knee disarticulations this study may not have the power to detect other key variables in determining postoperative ambulation. For example, all patients with end stage renal disease or a history of stroke were non-ambulatory following surgery, although these factors did not achieve significance. A large, multicenter, prospective study is likely needed to determine the significance of these trends that have not achieved statistical significance.

\section{REFERENCES}

1. Behr J, Friedly J, Molton I, et al. Pain and pain-related interference in adults with lower-limb amputation: comparison of knee-disarticulation, transtibial, and transfemoral surgical sites. J Rehabil Res Dev 2009;46:963-72.

2. Kulkarni J, Pande S, Morris J. Survival rates in dysvascular lower limb amputees. Int J Surg 2006;4:217-21.

3. Sansam K, Neumann V, O'Connor R, et al. Predicting walking ability following lower limb amputation: a systematic review of the literature. J Rehabil Med 2009;41:593-603.

4. Raichle KA, Hanley MA, Molton I, et al. Prosthesis use in persons with lower- and upper-limb amputation. J Rehabil Res Dev 2008;45:961-72.

5. Krajbich JI. Lower-limb deficiencies and amputations in children. J Am Acad Orthop Surg 1998;6:358-67.

6. Ten Duis K, Bosmans JC, Voesten HG, et al. Knee disarticulation: survival, wound healing and ambulation. A historic cohort study. Prosthet Orthot Int 2009;33:52-60.

7. Pinzur MS, Gold J, Schwartz D, et al. Energy demands for walking in dysvascular amputees as related to the level of amputation. Orthopedics 1992;15:1033-6.

8. Gailey RS, Wenger MA, Raya M, et al. Energy expenditure of trans-tibial amputees during ambulation at self-selected pace. Prosthet Orthot Int 1994;18:84-91.

9. Morse BC, Cull DL, Kalbaugh C, et al. Through-knee amputation in patients with peripheral arterial disease: a review of 50 cases. J Vasc Surg 2008;48:638-43.

10. Borkosky SL, Roukis TS. Incidence of re-amputation following partial first ray amputation associated with diabetes mellitus and peripheral sensory neuropathy: a systematic review. Diabet Foot Ankle 2012;3:12169.

11. Attinger CE, Brown BJ. Amputation and ambulation in diabetic patients: function is the goal. Diabetes Metab Res Rev 2012;28 Suppl 1:93-6.

12. Evans KK, Attinger CE, Al-Attar A, et al. The importance of limb preservation in the diabetic population. J Diabetes Complications 2011;25:227-31.

13. Taylor SM, Kalbaugh CA, Blackhurst DW, et al. Preoperative clinical factors predict postoperative functional out- 
comes after major lower limb amputation: an analysis of 553 consecutive patients. J Vasc Surg 2005;42:227-35.

14. Fisher DF Jr, Clagett GP, Fry RE, et al. One-stage versus two-stage amputation for wet gangrene of the lower extrem- ity: a randomized study.J Vasc Surg 1988;8:428-33.

15. Nellis N, Van De Water JM. Through-the-knee amputation: an improved technique. Am Surg 2002;68:466-9. 